\title{
PROCESS AND SYSTEMS Sort your waste! An audit on the use of clinical waste bins and its implications
}

\author{
Author: Harriet Runcie
}

This article reports a multicentre quality improvement project on the use of clinical waste bins and aims to raise awareness of the importance of sorting waste. It also makes recommendations for hospital clinical and management staff to improve compliance.

An audit on the contents of clinical waste bins at two NHS trusts was performed against hospital guidelines. Eighty clinical waste bins were spot-checked and their visible contents recorded.

In total, 347 items were seen in 80 bins; $59 \%$ of items were non-clinical, $40 \%$ clinical. The results were presented at a medical department meeting and to the hospitals waste management teams with suggestions for improvement. A re-audit on a test ward was then performed. No improvement was seen, with $66 \%$ of the waste found to be non-clinical, $31 \%$ clinical.

Therefore, the aims of this article are to educate clinicians and hospital staff and encourage waste separation to improve compliance on the use of clinical waste bins, resulting in savings in the environmental and financial costs of waste disposal.

KEYWORDS: Quality improvement project, audit, waste management, clinical waste, environment sustainability

\section{Introduction}

The management of waste in the UK is governed by legislation, which is defined by the European Commission's Waste Framework Directive, 2008. The Control of Substances Hazardous to Health (COSHH) Regulations and the Management of Health and Safety at Work Regulations, produce legislation particularly on the handling of potentially infectious or hazardous waste, which is based on assessment of risk to staff and the public. The Department of Health, in partnership with the Department for Environment, Food and Rural Affairs (Defra) has issued guidelines, '07-01: Safe management of healthcare waste,' based on these regulations. It is a legal requirement that waste is sorted and coded and then managed appropriately. Staff segregating waste should be provided with appropriate training and clear instructions on waste segregation and labelled, colour-coded waste receptacles are advised for each waste stream. The aim of waste segregation is to firstly comply with

Author: core medical trainee, Country Durham and Darlington NHS Trust, Darlington, UK the law, but also to reduce the risk of exposure and injury to those handling it, to improve waste minimisation and recycling, reduce energy requirements and associated carbon production and reduce financial costs. It ensures the waste is handled, transported and disposed of in a safe and effective manner. ${ }^{1}$

Clinical waste is defined by the Controlled Waste Regulations issued under the Environmental Protection Act and is defined as

a) any waste which consists wholly or partly of human or animal tissue, blood or other body fluids, excretions, drugs or other pharmaceutical products, swabs or dressings, syringes, needles or other sharp instruments, being waste which unless rendered safe may prove hazardous to any person coming into contact with it; and b) any other waste arising from medical, nursing, dental, veterinary, pharmaceutical or similar practice, investigation, treatment, care, teaching or research, or the collection of blood for transfusion, being waste which may cause infection to any person coming into contact with it.

Therefore, the majority of clinical waste is categorised as hazardous, posing either an infectious risk, a chemical risk or containing pharmaceutically active agents. It requires specialist handling and disposal and can either be treated at an appropriate licensed premises to render it safe prior to final disposal (using heat, chemicals or irradiation) or it can be incinerated. Black bin domestic waste is not hazardous and can be disposed of in landfill or by energy from waste processes (energy recapture). ${ }^{1}$ Clinical waste disposal is therefore performed at a higher cost to the environment, requiring more energy and producing more carbon emissions. ${ }^{1}$ It also has high financial costs. County Durham and Darlington NHS Foundation Trust spends roughly $£ 450,000$ per year on the disposal of waste, of which about $€ 350,000$ is clinical waste $(77 \%)$ and $£ 100,000$ other waste, including domestic, electrical and food (22\%). Waste disposal is charged per tonne, but as a rough average estimate, the disposal of 10 bags of clinical waste costs $£ 10$, compared to 10 bags of domestic waste at $£ 2.50$. (Estimates of costs provided in personal communication with Mr S Garth, Waste Manager, Estate Services, County Durham and Darlington NHS Foundation Trust.)

This article discusses an audit performed to investigate the use of clinical waste bins. It was identified as an important topic after it was noted that there was a difference in the management of clinical waste in New Zealand compared to the UK. In New Zealand most hospital staff are careful regarding what waste goes into which bin. There are always at least two bins to choose from: clinical or domestic, and often a third for recyclables. There are signs above and on the bins, with 
advice on which bin to use and information on the costs of processing each. In the UK, the author observed that bins are not conveniently sited, often a search is required to locate one, there is often only one option and most hospital staff do not seem to sort waste. For example, the author noticed in New Zealand people performing a simple clinical procedure like taking a blood sample, post-procedure and after disposing of any the sharps, would carefully sort through the equipment, removing any packaging and non-clinical items and putting them in the domestic bin, before putting the rest in the clinical bin. In the UK it was noted that most people after performing this procedure and disposing of any sharps tipped all the equipment into the clinical bin. Therefore, this audit was planned to assess the use of clinical waste bins in the UK and suggest improvements to improve the segregation of waste.

The audit focuses on clinical waste bins as these are present on all hospital wards. Other waste streams of importance are offensive waste, which is categorised as non-hazardous, but potentially offensive or unhygienic and can include incontinence and sanitary waste and medical equipment that does not pose an infection risk, and sharps, which have been discussed extensively previously. Offensive waste bins are not used on the wards at James Cook University Hospital or Darlington Memorial Hospital, so were not included in this audit. Also of importance are cytotoxic waste, radioactive waste and anatomical waste found in specialist areas, which are beyond the scope of this audit.

The article adds to what is already known about clinical waste management and demonstrates a need for action and education.

\section{Methods}

Over a period of 2 months a total of 80 clinical waste bins at James Cook Hospital, Middlesbrough and Darlington Memorial Hospital were audited. Guidelines used were the hospital guidelines on the use of clinical waste; only clinical waste is to be put in a clinical waste bin: gloves, aprons, dressings, swabs, continence pads, catheters/bags, items soiled with bodily fluids). No non-clinical waste including food, newspapers, paper towels, packaging, plastic containers, drink cans, aerosols, and particularly no sharps or glass (Fig 1).

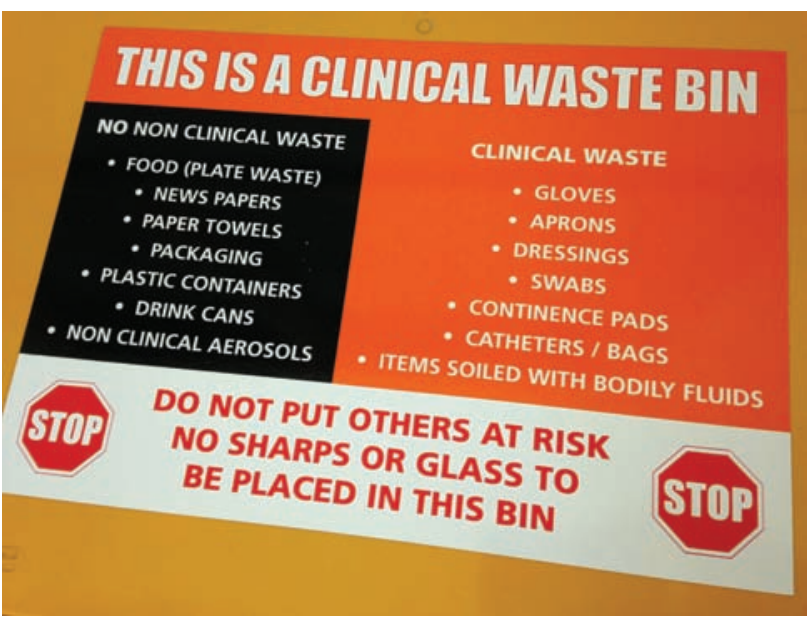

Fig 1. Photo demonstrating the hospital guidelines printed on a clinical waste bin lid.
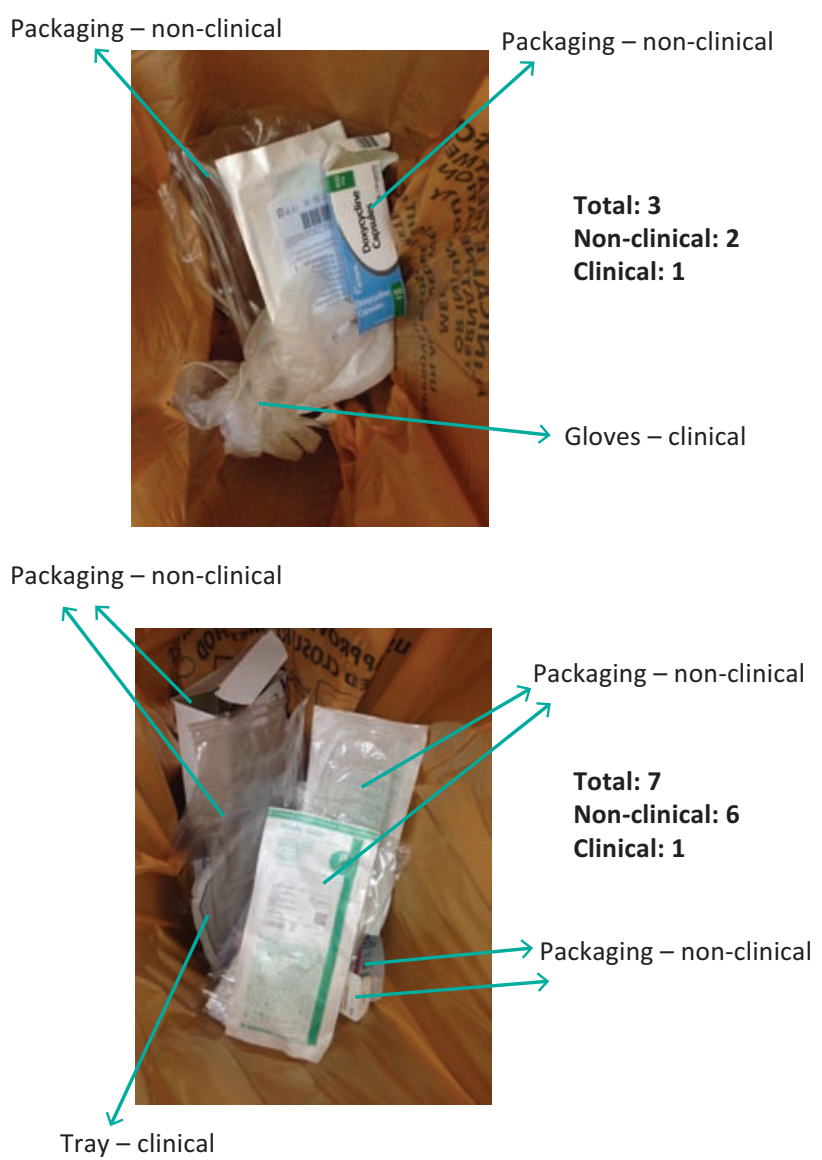

Fig 2. Photo demonstrating the spot audit of visible items on opening a clinical waste bin.

A spot check of 80 bins was performed over a variety of wards. The top of each bin was opened and the items visible were recorded as non-clinical, clinical or visibly soiled. Items that could have potentially been contaminated, such as disposable trays, were categorised as clinical (Fig 2). Any unidentifiable items were recorded but not categorised. This method was chosen as a simple, quick and easily reproducible way of assessing waste segregation and prevented recording any false habit changes that sometimes occur when people know they are being observed. This method is limited as only a snapshot of a stratum of the bin is observed as opposed to the total contents. Cost savings have not been calculated as this would require specialist training in waste handling so the bin contents could be weighed. Given that the aims of the audit were to assess waste segregation habits, to raise awareness, improve waste management compliance, and weighing the contents would impair future reproduction of the audit due to the extra complexity and additional time requirements, this was deemed unnecessary. As already detailed, offensive waste bins were not included, as they are not present on the wards of the two hospitals involved in this audit.

\section{Results}

In total, 347 items were seen in 80 clinical waste bins. Of these items, 206 (59\%) were identified as non-clinical, of which $96 \%$ was 


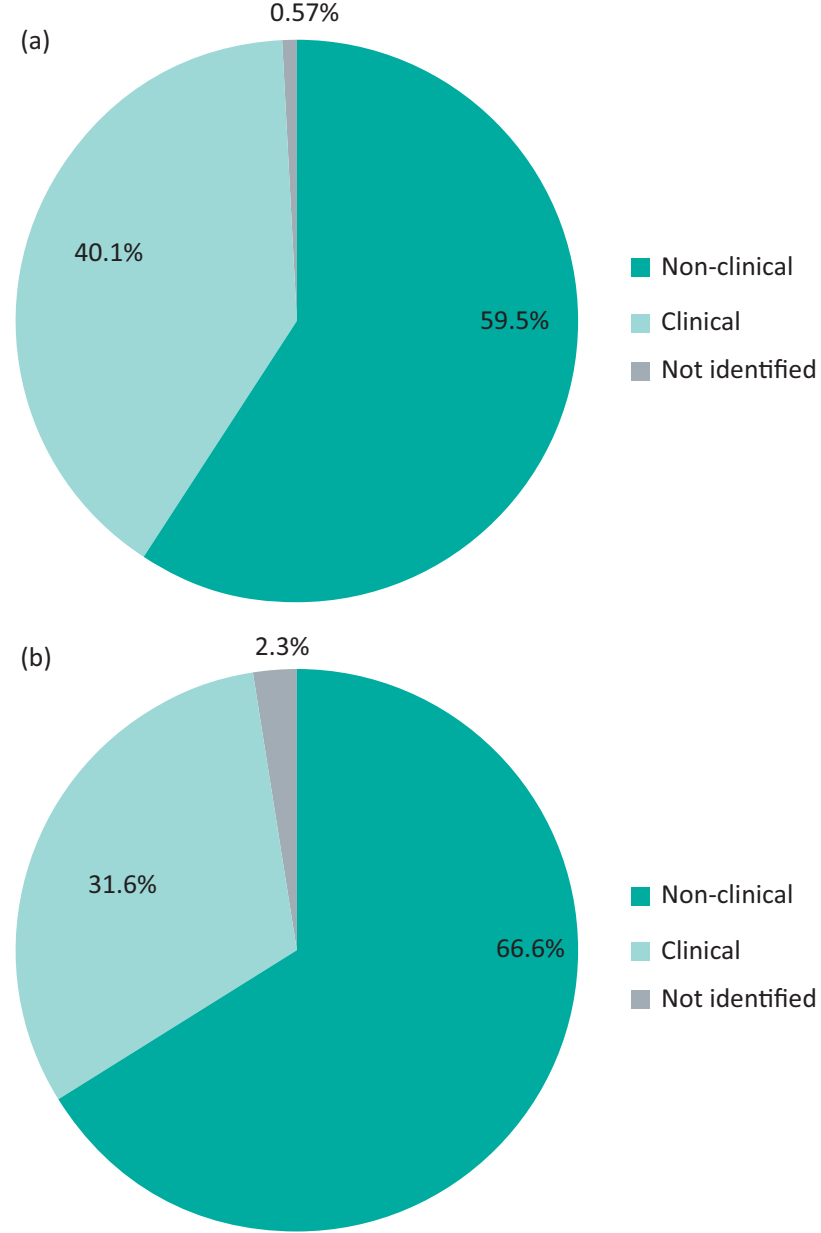

Fig 3. Pie chart demonstrating the results of initial and re-audit audits. (a) Percentage contents of clinical bins; (b) re-audit percentage contents of clinical bins.

packaging and $4 \%$ paper towels. Conversely, 139 items (40\%) were deemed clinical, composing of mainly aprons, gloves and disposable trays. Of the 139 clinical items, seven were visibly soiled. Two items could not be identified and so could not be categorised (Fig 3a).

The results of the audit were then presented at the medical department meeting and to the hospitals' waste management teams, and included recommendations for improvements. The County Durham and Darlington waste management team were initially interested and suggested a test ward to perform the improvement suggestions, including educating the staff on the ward, a review of the bin placement and siting posters to encourage waste separation. Unfortunately it was difficult to subsequently engage the team. The placement of bins was deemed difficult due to space constraints and despite encouragement the posters were not made. However, staff education was performed, following on from the medical department presentation, and staff on the ward including the full multidisciplinary team of doctors, nurses and physiotherapists were encouraged to segregate waste.

A re-audit was performed a month later. Bins on the test ward were surveyed and their contents recorded over a period of 2 weeks, until 40 bins were included. The results demonstrated no improvement, with $66 \%$ of the contents found to be non-clinical waste, $31 \%$ clinical and $2.5 \%$ unidentifiable (Fig 3b).

\section{Discussion}

The outcome of this audit was very poor, with only $41 \%$ of items meeting guidelines for disposal of clinical waste. This result is in keeping with other audits published. The Royal Berkshire Hospital produces approximately 480 tonnes of clinical waste a year but an estimated $40 \%$ of this is not clinical waste with items such as packaging, dead flowers, hand towels and newspapers all going into the clinical bins. ${ }^{1}$ Additionally, Hames audited clinical waste bins in an anaesthetic department in Melbourne, Australia, and found $44 \%$ was non-clinical waste, compared with $41 \%$ clinical. $^{2}$

Unfortunately it was difficult to engage both staff within management and clinical staff on the ward. Despite educational talks there was little change in behaviour and some of the suggestions following the first audit, such as a review of bin position and ensuring the availability to two bins next to each other, was not done.

Other articles have looked into how to improve waste management in hospitals. Hames noted that simple education of members of staff alone was not enough and suggested multiple approaches are required. ${ }^{2}$ Ezibe-Ejiofer et al demonstrated a $90 \%$ reduction in clinical waste in a scrub room and $50 \%$ in the anaesthetic room following a 3-week period of education, group briefings and posters. ${ }^{3}$ Ivory found that by adding a domestic waste bin next to a clinical waste bin at intensive care bedsides resulted in a $12 \%$ saving for the ICU. ${ }^{4}$ Jaladi and Corner found that by adding a recycling bin into an anaesthetic theatre about $10 \%$ of the waste was recycled each day. ${ }^{5}$ These articles demonstrate that simple measures can significantly reduce the amount of non-clinical waste that ends up in clinical waste bins but that there has to be multiple approaches and team engagement, and support from management is vital.

There are examples of successful waste management reviews that have led to financial savings. The Department of Health reports two. The first is a review of waste management performed by the University College London, which produces approximately 250 tonnes of healthcare and clinical waste per year. They performed a complete review of their waste management, including implementation of an offensive waste stream, introducing recycling bins, redesigning the waste collection schedule to reduce transport journeys and performing more energy from waste recapture, and reported savings of over $£ 100,000$ and a $24 \%$ reduction in their carbon footprint over a 3 -year period. 'This was achieved with the appointment of an on-site waste expert to design and implement the new approach. The second report is the success of the Royal Berkshire Hospitals Foundation Trust, which focused on staff education, creating a staff information pack on waste segregation, appointing 'Waste watcher champions' as a point of contact on each ward and introducing a session on waste management within induction training days for new staff. They identified a potential saving of $£ 71,040$ per year if clinical and non-clinical waste was disposed of in the correct bins and found that other benefits included improved safety for staff, cleaner environments and more motivated staff. ${ }^{1}$ Again this was achieved with a facilities manager overseeing and implementing the changes. Ezihe-Ejiofor's audit was performed in a hospital that spends $£ 340,000$ on clinical waste per year, similar to Darlington Memorial Hospital. Following Ezihe-Ejiofor's audit a potential saving in the hospital of $£ 150,000$ per year was suggested, although details on how this figure was obtained are not detailed. ${ }^{3}$ 
It is also of note that the two hospitals in this audit do not have offensive waste bins (usually labelled with yellow and black strips) on their general wards. Offensive waste includes items contaminated with bodily fluids (urine, faeces, vomit, sputum and blood) known to be not infectious. For example, urine bags, incontinence pads, nappies, maternity and sanitary products, swabs and dressings. If it has been confirmed that the patient poses no risk of infection these waste products can be categorised as non-hazardous and as such disposal is much easier and cheaper than for clinical waste and can be treated similar to domestic waste: sent to landfill, energy from waste processes, recovery or incineration. However, the risk of contamination in this waste stream is high. For example, if a patient had an as yet undiagnosed Escherichia coli urinary tract infection and their urine bags were placed in the offensive waste this would contaminate the whole bin and put workers processing the waste at risk. Introduction of this waste stream would also involve adding another bin to the collection. There would need to be a clinical waste bin, an offensive waste bin, a domestic waste bin and potentially a recycling bin next to each other. Clinical areas and treatment rooms are often small and finding space for them would be challenging. It also makes the whole process more complicated thereby increasing the risk of poor compliance. Therefore, hospitals need to weigh up the benefits and risks of introducing this stream as a means of improving waste segregation.

This audit has identified three key measures to improve waste segregation. These are staff education, the appropriate placement of bins so they are easily accessible next to each other and the appointment of a manager to implement the changes.

The following recommendations are advised.

> Hospitals engage in education of staff, providing information on what constitutes clinical waste and on the differences in cost of clinical compared to domestic waste disposal.

> Universities and medical schools provide the students with information and education and encourage segregation of waste from the start of their careers.

> Hospitals review the placement of bins to ensure suitability and easy access. For example, domestic waste bins, not clinical waste bins, are placed under sinks for easy disposal of paper towels.

> Hospitals ensure a clinical waste and a domestic waste bin are placed next to each other so disposal of items appropriate to each is easy and available at the same time.

> Hospitals display posters above the bins clearly defining what waste should go in which bin, the purpose of segregating waste and the possible savings (Fig 4).

$>$ Hospitals consider increased use of the offensive waste stream.

$>$ Hospitals appoint someone within the waste management team to oversee the above.

\section{Summary}

This audit has highlighted that the segregation of waste is poorly performed, with $59 \%$ of items seen in clinical waste bins found to be non-clinical. Encouraging habit change is difficult and requires a multipronged approach. Raising staff awareness is key; education is vital to achieve this, then to facilitate change the placement of bins should be carefully reviewed. Team engagement, including both clinical staff and management, is needed and a facilitator to oversee the recommendations should be appointed. An NHSwide policy needs to be adopted to make these improvements to

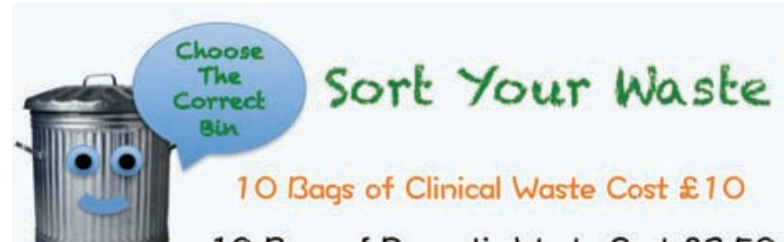

10 Bags of Domestic Waste Cost $£ 2.50$

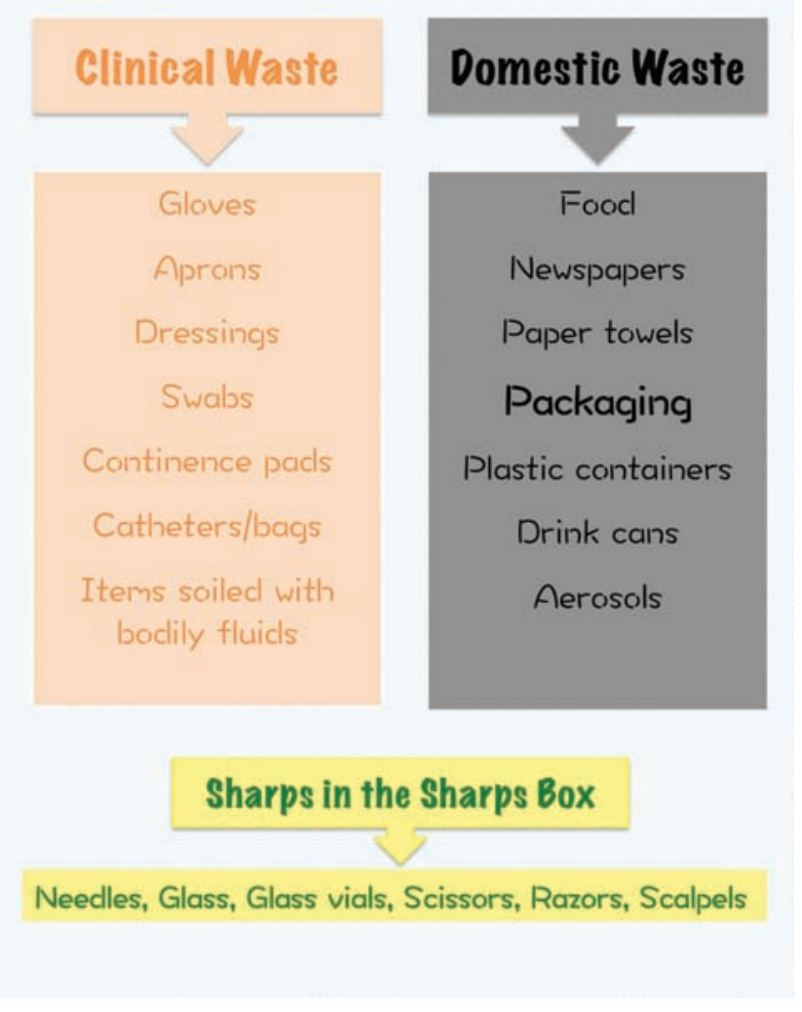

Fig 4. A suggestion for an educational poster.

achieve environment sustainability and significant cost savings for the NHS.

\section{References}

1 Department of Health. Environment and sustainability Health Technical Memorandum 07-01: Safe management of healthcare waste. DH, 2013.

2 Hames K. Healthcare waste disposal: an analysis of the effect of education on improving waste disposal. Healthcare Infection 2013;18:110-4.

3 Ezihe-Ejiofor JA, Hoque N, White SM. The 'Rubbish Project': Using data to drive change in the waste disposal culture in the operating theatres. Anaesthesia 2012;67(Suppl 2):20.

4 Ivory B. Cost savings achieved by the introduction of clinical and nonclinical waste bins in a United Kingdom ICU. Intensive Care Med 2011;37(Supp2):S34.

5 Jaladi S, Corner A. Anaesthetic waste disposal. Br J Anaesth 2011;107:286p-7p.

Address for correspondence: Dr Harriet Runcie, Microbiology Department, The Royal Infirmary of Edinburgh, 51 Little France Crescent, Edinburgh EH16 4SA, UK.

Email: h.runcie@nhs.net 